\title{
The Development of a Liver Abscess after Screening Colonoscopy: A Calculated Risk?
}

\author{
Simon Bac ${ }^{1}$, Dirk Jan Bac ${ }^{2}$ \\ ${ }^{1}$ Department of Gastroenterology, University Medical Center Utrecht, Utrecht, The Netherlands \\ ${ }^{2}$ Department of Gastroenterology, Gelderse Vallei Hospital, Ede, The Netherlands
}

Received: 02/05/2017

Accepted: 19/06/2017

Published: 04/07/2017

How to cite this article: Bac S, Bac DJ. The development of a liver abscess after screening colonoscopy: a calculated risk? EJCRIM 2017;4: doi:10.12890/2017_000650.

Conflicts of Interests: The Authors declare that there are no competing interests.

This article is licensed under a Commons Attribution Non-Commercial 4.0 License

\section{ABSTRACT}

We present the case of a patient who developed a liver abscess following screening colonoscopy. A colorectal screening program was introduced in the Netherlands in 2014 in order to reduce mortality from colorectal cancer. The patient in this report, a 63-year-old man with no significant medical history, underwent polypectomy of two polyps. Four days afterwards he presented to our emergency department with fever, nausea and vomiting. He was diagnosed with a Klebsiella pneumoniae liver abscess and was successfully treated with antibiotics for 6 weeks. This case highlights one of the risks of screening colonoscopy. Given the high number of colonoscopies due to the colorectal screening programs, we should be aware of complications in this mostly asymptomatic group of patients.

\section{LEARNING POINTS}

- Screening colonoscopy is a potential risk factor for Klebsiella pneumoniae liver abscess.

- It is thought that microperforations during colonoscopy could lead to bacterial invasion causing a pyogenic liver abscess.

- We expect the numbers of complications, including pyogenic liver abscess, to grow due to the increase in population-based screening programs for colorectal carcinoma.

\section{KEYWORDS}

(Pyogenic) liver abscess, Klebsiella pneumoniae, colonoscopy, screening

\section{CASE DESCRIPTION}

A 63-year-old man was scheduled for colorectal screening because of his age. He had atrial fibrillation, an inguinal hernia and a herniated nucleus pulposus. As an immunoassay faecal occult blood test (iFOBT) showed occult blood in his faeces, he underwent a screening colonoscopy. The colonoscopy revealed diverticulosis and three polyps, two of which were removed by polypectomy. The first polyp was located in the caecum and the second in the descending colon. The third polyp (Fig. 1), located in the hepatic flexure, was left in situ for endomucosal resection (EMR) in the near future. The patient returned home after colonoscopy. However, 4 days later he presented to the emergency department with fever, nausea and vomiting. His blood results showed a high C-reactive protein (251 mg/l, normal range 0-10 $\mathrm{mg} / \mathrm{I}$ ) and elevated liver enzymes (ALAT $169 \mathrm{IU} / \mathrm{l}$, normal range 0-35 IU/I). Sonography of the abdomen showed a mass measuring 5.7×4.6 $\mathrm{cm}$ in the right liver lobe. Computed tomography (CT) of the abdomen confirmed this liver abscess but showed no other abnormalities (Fig. 2). 
Blood cultures grew Klebsiella pneumoniae. The patient was treated with antibiotics for 6 weeks, after which the C-reactive protein was normalized and the abscess was no longer seen on sonography. The antibiotics were stopped and the patient underwent EMR of the third polyp performed under prophylactic antibiotics in a referral centre. This third polyp was benign, as were the other two polyps. All three were tubulovillous adenomas.

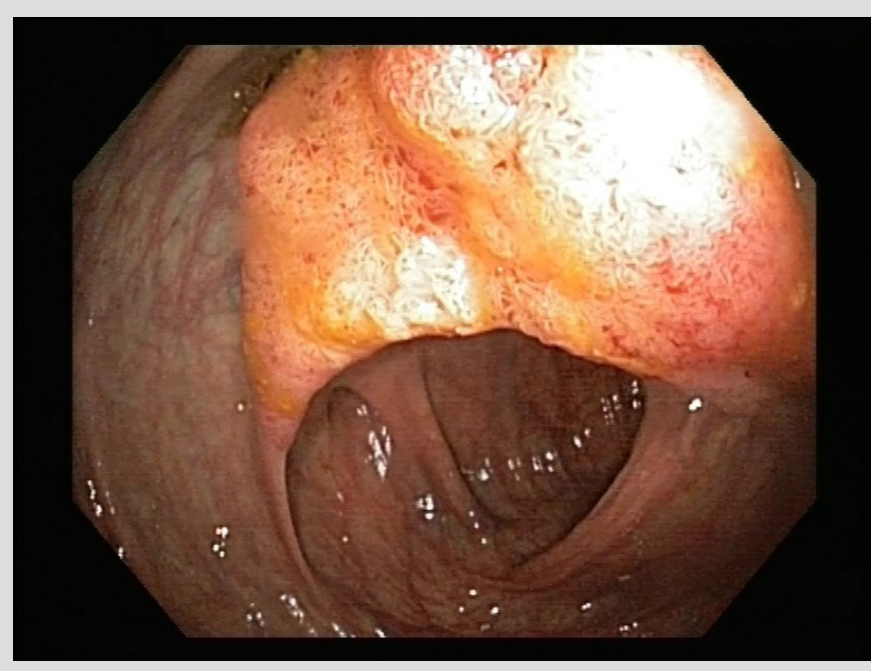

Figure 1. Polyp in the hepatic flexure

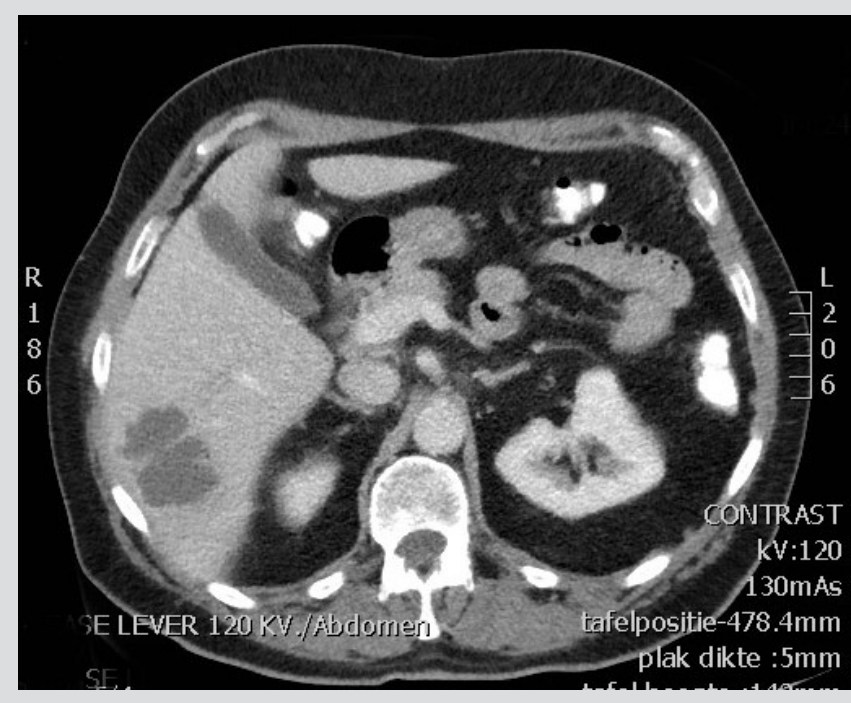

Figure 2. CT scan showing the liver abscess

\section{DISCUSSION}

A pyogenic liver abscess (PLA) is potentially life threatening, has a mortality rate is $5.6 \%$ in the USA and is increasing in incidence ${ }^{[1]}$. Klebsiella pneumoniae is the pathogenic microorganism in approximately $27 \%$ of PLA ${ }^{[2]}$. Other common microorganisms are the Streptococcus milleri group (44\%), Escherichia coli (16\%) and anaerobes (20\%). Risk factors for a K. pneumoniae liver abscess are diabetes mellitus and metastatic infection ${ }^{[3,4]}$. The patient in our case did not have these risk factors.

However, our patient did have tubulovillous adenomas and underwent colonoscopy. A few case reports suggest that tubulovillous adenomas are a risk factor for PLA, irrespective of the pathogenic microorganism ${ }^{[5,6,7]}$. Colonoscopy might cause mucosal defects at the site of the lesions providing a route for bacterial invasion of the portal system. Furthermore, there is a correlation between colonoscopy and the development of a PLA, although literature on the subject is scarce ${ }^{[8]}$, perhaps due to the difficulty in establishing a causal relationship between colonoscopy and liver abscess. A PLA after colonoscopy might be due to microperforations during colonoscopy, leading to bacterial invasion ${ }^{[6]}$.

A large retrospective study from Taiwan showed an increased risk for colorectal carcinoma in patients with a PLA caused by K. pneumoniae, compared to non-K. pneumoniae PLA patients ${ }^{[9]}$. Therefore a colonoscopy is advised, if it has not already been performed at K. pneumoniae PLA diagnosis.

In conclusion, we describe a patient with a K. pneumoniae PLA 4 days after undergoing screening colonoscopy with polypectomy. One should be aware of the potential risk of PLA following colonoscopy. The risk factors for PLA are not known but might include polyps, polypectomy or diverticular disease. Some $56 \%$ of all PLAs are defined as cryptogenic ${ }^{[2]}$, but we do not know how many may have occurred followed a colonoscopy as there is a lack of information. We recommend further research into the association between K. pneumoniae PLA and colonoscopy. Furthermore, we should expect increasing numbers of complications, including PLA, due to the growth in population-based screening programs. 


\section{REFERENCES}

1. Meddings L, Myers RP, Hubbard J, Shaheen AA, Laupland KB, Dixon E, et al. A population-based study of pyogenic liver abscesses in the United States: incidence, mortality, and temporal trends. Am J Gastroenterol 2010;105:117-124.

2. Kaplan G, Gregson DB, Laupland KB, Population-based study of the epidemiology of and the risk factors for pyogenic liver abscess. Clin Gastroenterol Hepatol 2004;2:10321038

3. Wang JH, Liu YC, Lee SS, Yen MY, Chen YS, Wang JH, et al. Primary liver abscess due to Klebsiella pneumoniae in Taiwan. Clin Infect Dis 1998;26:1434-1438.

4. Kamal F, Williams G, Akbar H, Khan MA, Kadaria D. Klebsiella pneumoniae liver abscess: a case report and review of literature. Cureus 2017;9:e970.

5. Lai HC, Chan CY, Peng CY, Chen CB, Huang WH. Pyogenic liver abscess associated with large colonic tubulovillous adenoma. World J Gastroenterol 2006;12:990-992.

6. Gross RG, Reiter B, Korsten MA. Pyogenic liver abscess complicating colonoscopic polypectomy. Gastrointest Endosc 2008;67:767-768.

7. Tan CB, Shah M, Rajan D, Lipka S, Ahmed S, Freedman L, et al. A solid organising cryptogenic liver abscess and its association with a colonic tubullovillous adenoma. BMJ Case Reports 2012. doi: 10.1136/bcr-2012-006431.

8. Bonenfant F, Rousseau É, Farand P. Streptococcus anginosus pyogenic liver abscess following a screening colonoscopy. Can J Infect Dis Med Microbiol 2013;24:e45-e46.

9. Huang WK, Chang JW, See LC, Tu HT, Chen JS, Liaw CC et al. Higher rate of colorectal cancer among patients with pyogenic liver abscess with Klebsiella pneumoniae than those without: an 11-year follow-up study. Colorectal Dis 2012;14:e794-e801. 